\title{
La vie des morts : jesuismort.com, entre biographie et nécrologie
}

The Life of the Dead: jesuismort.com, between Biography and Obituary

\section{Adeline Wrona}

\section{(2) OpenEdition}

1 Journals

Édition électronique

URL : http://journals.openedition.org/questionsdecommunication/2605

DOI : 10.4000/questionsdecommunication.2605

ISSN : 2259-8901

\section{Éditeur}

Presses universitaires de Lorraine

\section{Édition imprimée}

Date de publication : 30 juin 2011

Pagination : 73-90

ISBN : 978-2-8143-0084-2

ISSN : 1633-5961

\section{Référence électronique}

Adeline Wrona, «La vie des morts : jesuismort.com, entre biographie et nécrologie ", Questions de communication [En ligne], 19 | 2011, mis en ligne le 21 septembre 2015, consulté le 19 avril 2019. URL : http://journals.openedition.org/questionsdecommunication/2605; DOI : 10.4000/ questionsdecommunication.2605 


\author{
ADELINE WRONA \\ Groupe de recherche interdisciplinaire sur les processus d'information \\ et de communication \\ Celsa, Université Paris-Sorbonne \\ adeline.wrona@celsa.paris-sorbonne.fr
}

\title{
LA VIE DES MORTS : JESUISMORT.COM, ENTRE BIOGRAPHIE ET NÉCROLOGIE
}

Résumé. - «Tout discours sur les morts fonde les vivants », écrit Jean-Claude Bonnet (1997: | 83 I) dans les Lieux de mémoire. Guidé par cette proposition, cet article analyse une nouvelle forme d'écriture de la mort rendue possible par les médias informatisés. L'exemple choisi est celui d'un site intitulé « jesuismort.com», créé en 2005 par un développeur web qui renouvelle les formes de l'éloge post mortem en hybridant des modèles hérités avec les potentialités propres aux écrits d'écrans. Le discours tenu sur les morts détermine des gestes communicationnels qui donnent sens à l'hommage : lire une biographie, écrire une lettre, suggérer un mort, mais aussi améliorer le « score » de popularité des défunts célèbres, devenir « fan » du site sur Facebook. Tous ces possibles offerts par le site constituent autant de participations d'un nouveau genre à un rituel funéraire largement trivialisé qui est ici examiné selon trois directions successives: du point de vue de son régime temporel, de son modèle économique, enfin de ses modes de sociabilité.

Mots clés. - Biographie, écrits d'écran, présentisme, réseaux sociaux, monétisation, filiation inversée. 


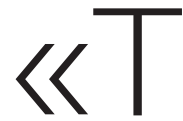

out discours sur les morts fonde les vivants », écrit Jean-Claude Bonnet (1997: 183|) avant de parcourir en historien ces « lieux de mémoire » textuels que sont les oraisons funèbres, et leurs déclinaisons en des genres voisins - éloges académiques, nécrologies, notices bio-bibliographiques, Gazette des deuils et autres Nécrologe des hommes célèbres. La presse, l'édition, les médias dans leur ensemble, édifient eux aussi des monuments du souvenir à ces « morts illustres » qui orientent les vivants au quotidien. À chaque support textuel correspondent des usages bien spécifiés qui reconfigurent les modes de l'hommage. Ainsi le développement de la presse périodique au XIX ${ }^{e}$ siècle offre-t-il un espace nouveau à la pratique ritualisée de la nécrologie qui se coule dans le rythme de la publication et offre au lecteur un panorama de la société adapté à ses intérêts supposés. Le discours sur les morts enregistre donc les mutations connues par les formes textuelles, qui engagent non seulement la nature des écrits eux-mêmes, mais aussi leurs modes de circulation et l'usage auquel ils se prêtent. À la manière de Jean-Didier Urbain (1989 : I0), on peut considérer le cimetière comme un lieu d'archivage des disparus qui passe par la production de textes. Aussi l'écriture a-t-elle fondamentalement à voir, au moins en Occident, avec la pratique du deuil et « le cimetière européen est à bien des égards, depuis le XIXe siècle, une bibliothèque ». Être un grand homme, se voir célébré comme un "mort illustre », ne correspond pas aux mêmes pratiques selon que l'hommage prend place à l'âge du papier ou de l'éloquence, des Lumières ou des sociétés sans histoires : « L'invention de l'imprimerie et de la poste », note encore Jean-Claude Bonnet (ibid. : 1834), « assure la conservation et la destination de tous les messages, si bien que le temps travaille pour le grand homme ». «Le cimetière des célébrités » : tel est le titre qui s'affiche sur la page d'accueil du site auquel nous allons consacrer notre analyse, jesuismort. com. Qu'advient-il du discours tenu sur les morts dans l'espace fluctuant des médias informatisés? La pierre des cimetières, le papier du livre ou du journal conservent les traces écrites déposées par les vivants pour perpétuer le souvenir des disparus. En revanche, le texte numérique ne vieillit pas avec son support si bien que «l'écran fait de l'écrit un objet éminemment fragile », comme l'écrit Emmanuel Souchier (1996: II5). Qu'est-ce qu'un cimetière informatisé et mobile, comme celui que propose jesuismort.com? Comment les morts nous apparaissent-ils au gré de ces écrits fugaces et sans cesse actualisés qui circulent sur les réseaux? Comment les formes du recueillement se reconfigurent-elles à l'aune d'une automatisation du recueil de données? Ce sont les questions que nous allons aborder en soumettant cet étrange objet de mémoire à trois analyses croisées qui, tour à tour, interrogeront le régime temporel de cette écriture commémorative, son modèle économique et, enfin, les gestes relationnels qui y sont favorisés par la mise en réseau et l'automatisation des échanges. La méthodologie utilisée consiste en une approche sémiotique des textes publiés sur ce site, faisant une large place à l'analyse des architextes informatisés; elle est complétée par un entretien mené avec le gestionnaire de jesuismort.com, joint par téléphone grâce aux traces nombreuses qui signent sa présence dans les marges des nécrologies. 


\section{Les morts actualisés : entre présentisme et filiation inversée}

C'est une promesse bien inquiétante que formule le titre choisi par ce site : « je suis mort », soit une phrase qu'il est impossible de prononcer et qui constitue le fantasme ontologique de tout écrivain, peut-être même de tout écrivant. Écrire sa mort, au passé et à l'accompli, quel coup de force rhétorique! Au moment de conclure le cycle des Rougon-Macquart, Émile Zola s'autorise ce geste prométhéen, en l'attribuant à son double romanesque, le Docteur Pascal : ce médecin qui ne tient qu'à un fil à la famille dont il s'est fait l'analyste (de son nom complet, il est Pascal Rougon) s'offre le privilège d'inscrire, quelques jours avant de mourir, la date de sa propre disparition sur l'arbre généalogique. Autoanalyste de sa maladie cardiaque dont il décrit la progression « en direct », il est aussi l'auteur de sa propre épitaphe. Vivre la mort en direct, écrire sa mort, archiver les éléments de son « portefeuille d'identité » numérique pour préparer sa postérité. De telles actions sont rendues possibles sur le réseau par toute une série de sites qui exploitent parfois sans vergogne le marché de la vie après la vie, tirant parti de la faiblesse institutionnelle et législative en ce domaine. En l'occurrence, le titre vaut pour jesuismort.com comme une accroche quasiment publicitaire : à qui « entre » sur le site, s'ouvre non pas un espace d'écriture de soi comme défunt, mais un lieu de commémoration des « morts célèbres ». Selon l'expression de Vladimir Jankélévitch, la « mort à la première personne » n'est qu'un effet d'annonce car c'est à ce grand « Autre », l'illustre disparu, que se consacre ce lieu de mémoire informatisé. Voici en effet le premier paragraphe de la page d'accueil :

LE CIMETIÈRE DES CÉLÉBRITÉS.

Biographie et célébrité disparue.

Entrez et venez vous recueillir sur la tombe des hommes et des femmes célèbres. Savourez I'histoire de chacun en consultant la biographie de chacun à moins que vous ne préfériez leur rendre hommage et faire ainsi évoluer leur score de popularité.

En toute rigueur, la mort n'est donc ici écrite ni à la première personne, ni au présent. Au-delà de l'effet provocateur du titre - tout à fait assumé par le créateur et gestionnaire du site (Branchut, 2010) -, cet espace consacré aux « morts illustres » a pourtant bien à voir avec l'actualité ou, au moins, avec ce processus de « mise à jour » des pages qui impose sur l'internet un régime d'actualisation automatisé. Mais de quelle actualité s'agit-il au juste? Tout d'abord, jesuismort.com n'échappe pas à la loi du flux, fortement dérégularisante, qui vaut pour toute publication en ligne: si le souvenir des disparus suscite des gestes commémoratifs ritualisés, l'inscription de ce passé dans le présent ne va plus de soi, une fois transposé sur l'internet. En effet, comment définir le « présent » sur le web quand tout est soumis à la promesse d'un renouvellement en continu? En ce sens, le média numérique exerce un effet de dépériodisation qui suppose la mise en œuvre de repères temporels d'autant plus solides: l'ethnologue américain Pablo Javier Boczkowski (2010:53) note que « la publication » d'un 
site d'information argentin, « devient une activité continue, sans fréquence déterminée, et sans rythme prévisible ». Dans le cas qui nous occupe, le point focal à partir duquel se déroule le discours tenu sur les morts se trouve dans un temps strictement calendaire. Sans se présenter comme un média du quotidien, jesuismort.com définit l'aujourd'hui par un recours au calendrier. Aussi l'hommage aux morts s'organise-t-il selon une logique proche de celle des vies de saints à qui l'on distribue un jour de l'année. Le prénom porté par la figure ainsi honorée scande, dans les calendriers traditionnels, le temps de la vénération collective organisant l'éternel retour du quotidien. Chaque jour, jesuismort.com propose un mort célèbre; son nom figure en page d'accueil, accompagné d'une série de portraits, graphiques, photographiques, ou vidéo. Métaphore littérale du cimetière, une pierre tombale porte l'un de ces portraits, encadré par plusieurs chiffres - au-dessus de son visage, ses dates de naissance et de mort, ainsi que son « classement », au-dessous, son « score ». Par exemple, le 13 juillet 2010, Arnold Schönberg figure à l'ordre du jour (voir illustration ci-dessous).

Figure I : L'anniversaire de la mort d'Arnold Schönberg ( 3 juillet 20 I0).

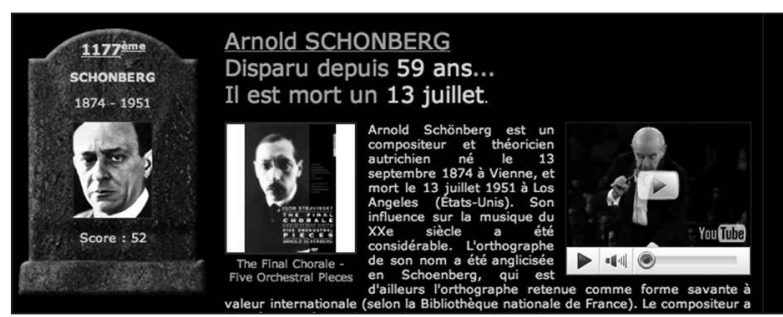

Le lien entre le visiteur d'aujourd'hui et ce mort célèbre réside dans la date portée par le calendrier : le 13 juillet, anniversaire de la mort du compositeur qui est aussi le jour de la visite.

Un outil algorithmique mis au point par le gestionnaire du site assure le calcul qui permettra de toucher du doigt le temps qui sépare le grand mort de l'internaute contemporain : comme on le voit sur la capture d'écran, l'intervalle nous séparant du disparu (59 ans) précède même la mention de la date du jour. C'est donc bien le présent - ce temps vécu collectivement par les vivants - qui tient lieu de repère vis-à-vis de la cohorte des grands hommes. Cet « âge » de la disparition n'a de sens qu'au moment de la consultation. De ce fait, la mise en ordre des morts célèbres reflète un principe d'organisation qui obéit à ce que Jean Davallon (2006: 74) appelle la filiation inversée, à savoir, le fait que « nous regardons les événements et les caractéristiques formelles dont les monuments sont porteurs moins à partir de leur origine que d'un cadre d'interprétation lié aux effets qu'ils produisent sur nous ». En d'autres termes, c'est l'ici et maintenant de ce « nous » qui fonde l'invention des morts célèbres comme patrimoine partagé : «Nous les abordons depuis notre point de vue, depuis notre présent » (ibid.). Jesuismort.com propose une série d'outils qui matérialisent cette organisation 
symbolique du passé à partir du présent: tous sont disponibles à partir de l'onglet « anniversaire », illustré par une icône de bougie allumée. Cette flamme, éphémère et fragile, donne la loi de fonctionnement de la commémoration numérisée, l'éternel recommencement de l'actuel dont le calcul d'intervalles automatise la mise à jour. Les « morts célèbres » viennent à nous selon un processus de patrimonialisation calendaire et automatique à la fois. Leur « valeur d'ancienneté »), selon l'expression reprise par Jean Davallon (ibid.) à Aloïs Riegl, est explicitement manifestée par cet « âge » dont nous fêtons l'anniversaire - un âge non pas biographique, mais qui mesure l'intervalle nous séparant de leur apparition ou de leur disparition. En ce sens, parler de la mort c'est surtout parler de la vie, celle qu'aurait pu vivre la célébrité dans cet espace de temps qui nous sépare de lui, celle qu'il ou elle a vécue, entre la date de sa naissance et celle de sa mort. La confusion des anniversaires, entre naissance et mort, signale en toute clarté la mise en équivalence de ces deux événements dès lors qu'ils sont appréhendés depuis le présent. De même, ce site qui s'annonce comme un « cimetière des célébrités 》 distribue surtout des biographies: il retraite des contenus fournis sur d'autres espaces de publications mis gratuitement en circulation et qui n'ont pas la mort pour objet. L'essentiel des textes repris en notice pour chaque mort du jour consiste de fait en un recyclage des articles publiés sur Wikipédia. L'encyclopédie « coopérative » croise, pour une part, le projet de jesuismort.com dans la mesure où elle multiplie, elle aussi, les entrées nominatives. Dès lors qu'un biographé disparait, il peut prétendre à passer d'un support à un autre, du dictionnaire participatif au cimetière des célébrités. La collection des morts célèbres offerte sur jesuismort.com répond bien à un objectif patrimonial selon la présentation qu'en donne Yves Jeanneret (2008: 188) en indiquant que la patrimonialisation consiste « en un processus qui identifie certains objets particuliers comme étant capables de manifester au présent un passé disparu et se traduit par la création de dispositifs institutionnels et gestionnaires spécifiques ». En ce sens, il s'agit moins d'un cimetière que d'un musée. Toutefois, la nature même du média numérique invite à repenser ces termes : si les collections ont une histoire - celles de leur ouverture au public, des politiques d'acquisition ou des stratégies de mécénat -, l'exposition des morts célèbres sur l'internet présente un autre régime de temporalité. En effet, le processus de « mise en patrimoine » quotidien s'y voit complété par une réorganisation permanente des hiérarchies et des classements qui distribuent les biographies au jour le jour. La logique de la filiation inversée se complète d'un autre mode de fonctionnement, qui est celui du classement exploitant les outils de mesure si efficaces sur l'internet. La « métrique du web » (Dagiral, Parasie, $2010: 35)$ se décline ici en « scores 》 et « rangs 》 attribués à chaque célébrité défunte. Ces deux mesures de la célébrité résultent de calculs automatisés liés, à la fois, à la fréquentation des «tombes» proposées à l'internaute et à un curieux jeu de hasard: quand il parcourt la page entière consacrée par le site au grand homme défunt, le visiteur se voit proposer « d'améliorer son score », en cliquant sur une carte à jouer retournée. Celle-ci révèle alors un quotient multiplicateur $(\times 10, \times 20 \ldots)$, qui augmente, le lendemain, le score déjà totalisé par 
la figure en question. De ce fait, la « collection » des morts célèbres n'est pas seulement mouvementée par le passage des jours et le feuilletage des pages d'un almanach numérisé. À chaque visite, les morts se déplacent, recomposant la grille de visages qui, sur la marge droite de la page d'accueil, symbolise le «Top 50 » des morts les plus populaires.

Figure 2 : Le «Top 50 » des morts les plus populaires.

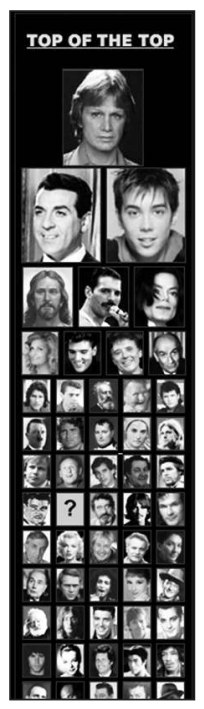

On le sait, toute lecture devient écriture dans le régime du numérique puisque, « à l'écran, on accède au texte par un acte d'interprétation » (Jeanneret, Souchier, 1999 : 100). Lire les biographies des morts célèbres dans jesuismort.com, c'est aussi réécrire leur position dans la collection, et même participer fort activement à la redéfinition de leur célébrité. La nature même de cette patrimonialisation des « tombes » virtuelles s'en voit considérablement modifiée : le rythme quotidien, artificiellement choisi pour l'ergonomie générale du site, est concurrencé et déstabilisé par l'action des internautes et la nature relativement aléatoire de leurs déambulations. Parmi les défunts figurant à la une de jesuismort.com, la plupart n'ont pas attendu le développement des médias informatisés pour devenir célèbres: ni Jésus de Nazareth ( $10^{\mathrm{e}}$ le 23 juillet 2010 ), ni Rabelais $\left(800^{\circ}\right)$, ni Adolf Hitler $\left(24^{\circ}\right)$ ne doivent leur popularité à l'invention de ce dispositif de vote numérique. En revanche, leurs scores constituent la matérialisation d'une échelle de valeur qui ne reflète au bout du compte qu'un calcul robotisé, effectué à partir de gestes enregistrés par l'architexte. Alors même qu'il est simple choix de lecture, le clic est interprété comme un acte positif d'évaluation. La désignation comme « exemplaires » des « vies » dont on souhaite s'inspirer passe ici par un indice de fréquentation, d'ordre purement quantitatif.Aussi, dans le cadre de l'écran appelé par le visiteur, l'actualité des morts célèbres équivaut-elle à leur « présence »: 
plus le parcours de l'internaute sollicite leur image et la mise en visibilité de leur biographie, plus leur célébrité s'accroît, si bien que, en fin de compte, c'est le présent qui fait la loi, pierre d'achoppement définitive dans une encyclopédie hétéroclite composée par des visages venus de tous horizons. Le discours sur les morts, confirmons-le, fonde les vivants; ou même, ce sont les vivants qui font venir à eux les morts, dans un geste numérique qui n'est pas loin d'évoquer le spiritisme. En ce sens, les célébrités mortes mises en série sur jesuismort.com parachèvent la tendance au « présentisme » qui caractérise la figure des stars, selon l'analyse de François Hartog (200I) dans sa préface aux Vies parallèles. Comme les « grands hommes » ou les « hommes illustres », les stars offrent, à en croire l'historien, des modèles de vie, proposant un répertoire d'exemplarité. Toutefois, alors que « l'homme illustre se profilait sur un horizon de perfection révolue », se coulant dans « une économie du temps qui était celle de l'historia magistra », 《la star, elle, est emportée par le temps », venue « uniquement du présent, elle s'y consume, voire s'y abîme » (ibid. : 46). Le discours sur les morts alimente donc le goût du présent, et le dispositif mis en œuvre par jesuismort.com manifeste tous les symptômes de cette « crise du temps » à laquelle François Hartog (2003 : 223) donne le nom de « présentisme », ce phénomène qui veut « que l'on s'intéresse au passé non pas pour lui-même (ce qui est l'historicisme), mais en ayant en vue le présent ». Les « crises de temps » ont elles-mêmes une histoire et les discours tenus sur les morts en donnent bien des clés. À travers les reconfigurations narratives des «vies achevées », se donnent à lire les modes de transmission du passé au présent, et donc la prise en charge des héritages mémoriels. À en croire François Hartog (2001 : 47), il existe un lien entre le développement des médias de masse et le basculement dans un nouveau régime d'historicité : « L'économie médiatique produit, consomme et consume "les héros du jour" ou d'un jour ». Les morts médiatiques se « consomment », de fait, toujours au présent : telle est la loi imposée au discours « trivial », pour reprendre le terme d'Yves Jeanneret. L'intensification de la circulation textuelle qui fait la puissance des médias informatisés a pour contrepartie la confusion des espaces de publication. L'analyse du modèle de financement mis en place par jesuismort.com explicite les termes de cette évolution.

\section{Le commerce des morts}

Qui finance jesuismort.com? Comment le/les gestionnaires du site trouvent-ils le temps et les ressources nécessaires à l'animation quotidienne d'un architexte complexe? Ces questions se posent en parallèle d'une série d'observations empiriques: en marge du texte principal, le visiteur du site ne peut manquer d'être frappé par la prolifération des liens marchands qui dirigent vers des sites de commerce en ligne. Par ailleurs, les liens hypertextuels font apparaître de curieuses annonces publicitaires quand on attendrait des compléments d'information concernant les biographies déroulées. Le présentisme analysé précédemment 
donne à comprendre cette étrange monétisation à laquelle se prête la nécrologie sur jesuismort.com. Célébrer un mort, Jean-Didier Urbain (1989 : 106) l'a montré, c'est partager au présent quelque chose qui appartient au défunt: les rituels funéraires visent à « nier la séparation », en maintenant « le mort au sein de la quotidienneté sociale ». Au Mexique, ce « commerce imaginaire » va jusqu'à la consommation de confiseries macabres : la commémoration célèbre le mort tout en s'en débarrassant. L'acte même de consommer ne saurait se vivre qu'au présent. Aussi le développement d'une culture de masse, et plus largement de la société de consommation, constitue-t-il pour François Hartog (2003 : 16) l'une des raisons qui expliquent le présentisme : le présent, expliquet-il en citant Hannah Arendt, est la « cible » de la société de consommation, sa « raison sociale ». Quotidiennement, ce grand magasin des morts célèbres qu'est jesuismort.com place en vitrine un homme du jour; les formes de célébration proposées à l'internaute combinent actions éditoriales « gratuites » et actes d'achats, selon une logique propre à l'univers de ce que l'on appelle parfois le « web social » qui fait de «l'agrégation des contenus postés par les utilisateurs 》 la « première source de valeur économique » (Millerand, Proux, Rueff, 2010 : 16). Sur le plan commercial, jesuismort.com adopte les ressorts de ce modèle économique, pratiquant notamment de façon systématique le « remixage » (ibid.: 5). Le site exploite des ressources non soumises aux droits d'auteurs, créant ainsi de la valeur par leur combinaison inédite. Ce fonctionnement est bien connu des spécialistes du marketing numérique; reste à en interpréter les conséquences, d'ordre symbolique notamment, dans le cas particulier de la nécrologie en ligne. Le premier effet de cette économie du « remix » réside dans l'emprise exercée par le réseau sur les prédilections éditoriales. De fait, la publication s'avère surdéterminée par les stratégies commerciales des éditeurs de texte. La biographie des célébrités est reprise sur Wikipédia, qui pratique un système de licence libre (GFDL). De même, quand la figure commémorée s'y prête, le site reprend des contenus vidéo mis en ligne sur Youtube. Finalement, au-delà de l'adresse liminaire citée plus haut, jesuismort.com ne produit qu'un très petit nombre de contenus en propre. En assurant une fonction ambivalente de conservation des morts et d'invitation à leur « consommation », le site se fait moins producteur de contenu qu'agrégateur, hybridant des fonctions souvent confondues dans les écrits de réseau. En l'occurrence, une telle posture s'explique aussi par les choix stratégiques de son gestionnaire avec lequel nous avons pu entrer en relation. Commençons par décrire les trois formes de financement mises en visibilité sur les pages du site jesuismort.com - les manifestations sémiotiques de ce marketing du lien méritent en effet d'être analysées en propre. La première source de monétisation réside dans un partenariat avec Amazon : le mort du jour se décline sur la marge de droite en une série d'objets à offrir en « cadeaux ». Selon le principe des « liens-produits », l'icône du paquet enrubanné dirige systématiquement vers une page du site marchand. Le nom du mort célèbre, repris en complément de sa biographie, devient littéralement un signe passeur vers des espaces de commerce en ligne. II en va ainsi de Victor Hugo dont la mort, en 1885, avait déjà suscité une effervescence commémorative fort 
lucrative (Ben Amos, 1997) et qui se voit recyclé en toute une série de « produits dérivés ». À l'amateur du poète qui consulte sa biographie sera proposé de façon aléatoire un extrait vidéo en noir et blanc sur lequel un acteur à la ressemblance stupéfiante récite le poème le plus connu des Contemplations (« Demain, dès l'aube »), ou bien des livres à acquérir « sur un click» par Amazon et d'autres produits bien plus éloignés du champ d'intérêt supposé de l'internaute. Ainsi, au cours de deux visites-test successives, à la recherche de la «tombe » de Victor Hugo, les « idées cadeaux 》 suivantes nous ont-elles été proposées:

Figure 3 : Des idées cadeaux en lien avec la visite du site.
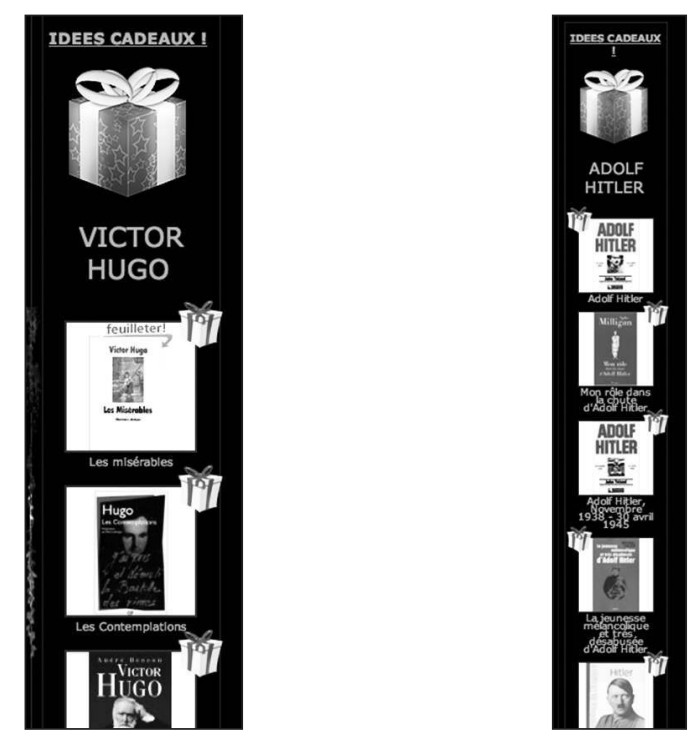

Entre Victor Hugo et Adolf Hitler, pas d'autre relation que celle établie par la « pêche » aléatoire des liens commerciaux. La seconde forme de financement consiste en des liens publicitaires qui obéissent eux-mêmes à deux logiques distinctes: d'abord, le site affiche des liens publicitaires proposés par Google Adsense. Ainsi les espaces entourant les biographies proposent-ils de rediriger le visiteur vers d'autres sites que le système robotisé a jugé susceptibles de l'intéresser: « visites de lieux saints », « calcul du jour de son décès » (www. jourdedeces.fr), mais aussi, pour qui veut lire la vie de Lady Diana, « hôtel Lady Diana à Istanbul », l'éventail des parcours proposés ne laisse pas de ménager quelques surprises savoureuses. Le summum de l'incongruité est atteint avec le second système de liens sponsorisés confié par le gestionnaire du site à une régie en ligne qui se charge d'insérer des annonces dans les termes même du texte publié. Quand la souris s'y attarde, les liens hypertextuels font surgir des objets à acquérir en un geste. De ce fait, à qui consulte par exemple la biographie d'Edmond de Goncourt est proposé un « remix» - sans les intertitres, ni les encadrés, et dans une mise en page adaptée - de la biographie mise en 
ligne sur Wikipédia. La rééditorialisation du contenu produit par l'encyclopédie participative s'opère à la fois par le nivellement général du texte, privé de ses niveaux hiérarchiques originaux pour être fondu dans le moule imposé par le nouveau dispositif éditorial et par l'insertion de liens commerciaux hypertextuels. D'où les effets d'incohérence ou d'incongruité du passage suivant : reprenant le paragraphe consacré sur Wikipédia au « Journal »d'Edmond de Goncourt qui s'organisait en une énumération des éléments identifiés comme composant les mémoires des deux frères, le propos ici réintégré dans le continuum d'un récit biographique livre un texte décousu, d'où se détachent nettement, comme des balises errantes, les termes isolés pour des raisons commerciales.

Figure 4 : Texte publié sur la page « Edmond de Goncourt » du site Wikipédia, http ://fr.wikipedia.org/wiki/Edmond_de_Goncourt.

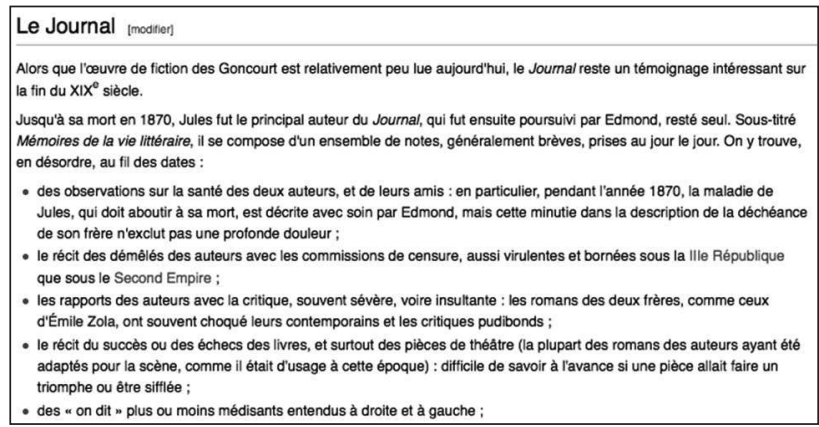

Figure 5 : Copie d'écran de la page consacrée à Edmond de Goncourt sur jesuismort.com.

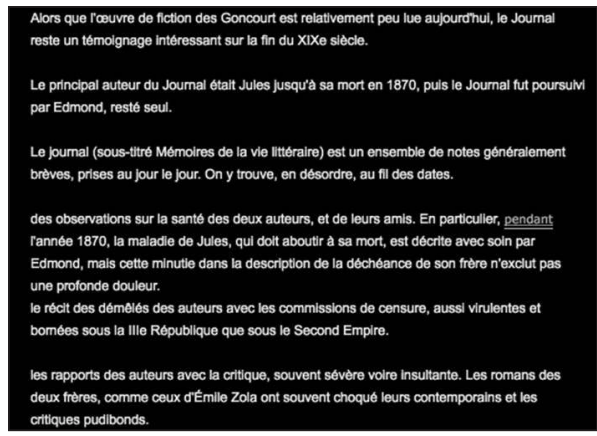

Un lien est proposé sous le terme « pendant ». Un clic fait alors apparaître un objet surprise, comme dans une tombola surréaliste - ici un produit de coiffage qui protège les cheveux pendant le séchage. Pour finir, le site s'inscrit dans un certain nombre de partenariats mentionnés en bas de page. Cette fois, il ne s'agit pas d'annonces payantes, ni même de liens sponsorisés, mais d'un système d'échange de liens voué à générer le plus de trafic possible entre sites partenaires. La liste 
en est particulièrement longue, surtout dans sa version complète, qui n'est pas affichée d'emblée sur la page d'accueil. Au-delà du caractère hétéroclite de ces partenariats, plusieurs catégories sont surreprésentées: les annuaires, les sites d'information « people », les sites ludiques, tout ce qui a trait à la mort en ligne, enfin des agences de développement web. Ce dernier domaine de compétence donne une clé importante à la compréhension de ce site nécrologique. Intriguée par la multiplicité des renvois vers ces sites de techniciens du web, nous avons fini par identifier le gestionnaire de jesuismort.com, qui se livre dans cette liste de partenariats à une autopromotion plus ou moins discrète. Développeur web, le responsable de ce site a accepté de répondre à nos questions dans un entretien téléphonique, ce qui nous a permis de mesurer l'enjeu éditorial qui est celui d'une telle publication. Le principal enseignement de cette conversation est le suivant : pour son éditeur, jesuismort.com représente une sorte de laboratoire, où il expérimente les outils de développement commercialisés par son agence web, « Studio vitamine ». Le créateur et gestionnaire de notre objet d'étude se décrit lui-même comme un technicien du web qui s'est initié de façon spontanée à la publication de contenu avec l'aide d'un directeur artistique. Or, comme l'indique la page d'accueil, la spécialité revendiquée par son agence réside précisément dans la « création de sites internet et référencement 》, d'où la faiblesse relative des textes inédits sur le site et la prolifération des liens sponsorisés ou commerciaux. Non que jesuismort.com vise à enrichir son éditeur - la monétisation lui rapporte, estime-t-il, tout juste de quoi financer l'hébergement du site - mais ce « hobby » d'un genre plutôt rare lui permet de tester l'efficacité et la pertinence des systèmes proposés à ses clients. Au bout du compte, le « cimetière des célébrités » en ligne s'apparente à un laboratoire de liens, non seulement parce qu'il mêle la dimension encyclopédique (le texte liminaire s'adresse aux 《 assoiffés de culture ») et l'ingéniosité commerciale mais, surtout, parce qu'il suscite la mise en relation d'une multitude d'individus qui, vivants ou morts, n'auraient jamais dû se rencontrer. Fort de ses 10000 à 15000 visiteurs quotidiens, jesuismort.com prouve à quel point la question du « stock des morts », pour reprendre une expression de Jean-Didier Urbain, se voit renouvelée par le pouvoir des architextes. Rappelons qu'au XIXe siècle, le marché des biographies a suscité un engouement comparable et phénoménal parmi les acteurs de la chaîne du livre. Éditeurs, mais aussi imprimeurs, libraires, marchands de gravures, tous ces acteurs de la diffusion et de la publication des textes ont sollicité, autour de 1840, les auteurs susceptibles de répondre au goût du public pour la vie des contemporains : écrivains, journalistes (Eugène de Mirecourt), auteurs de dictionnaires, photographes (Nadar), nombreux sont les professionnels de l'écriture qui se sont alors livrés à la pratique biographique. Dans son entreprise de commémoration des célébrités disparues, le créateur de jesuismort.com donne à lire quelque chose du goût contemporain pour la mort vécue au présent. Acteur de la chaîne de publication, mais non auteur des textes mis en ligne, ce technicien de la diffusion propose un dispositif éditorial adapté aux enjeux des médias informatisés; en ce sens, le programmateur s'impose comme un acteur clé de l'énonciation éditoriale. Comme nous allons le voir dans 
un troisième et dernier temps, le geste de publication y est inséparable de la mise en relation automatisée.

\section{Les vivants et les morts : commémorer, communiquer}

«Entrez et venez vous recueillir sur la tombe des hommes et des femmes célèbres. Savourez l'histoire de chacun en consultant la biographie de chacun à moins que vous ne préfériez leur rendre hommage et faire ainsi évoluer leur score de popularité ». Reprenons ce texte liminaire. L'acte de commémoration des morts célèbres s'y décline en une série de gestes qui sont autant d'implications de communication. L'impératif utilisé pour la série de scripts actionnels ici énumérés (《 entrez », « venez vous recueillir », « savourez », etc.) correspond tout à fait à cette « pente dans laquelle l'interaction s'engage si les sujets sociaux suivent sans distanciation la logique des formes auxquelles les productions médiatiques les confrontent » (Jeanneret, 2008 : I55). De façon plus générale, et au-delà même de ces lignes programmatiques, le site favorise le développement de relations qui s'inscrivent sur trois plans possibles : relations entre les vivants et les morts, relations entre vivants et vivants, mais aussi, si surprenant que cela puisse paraitre, relations entre morts et morts. Ces trois plans interagissent en permanence et d'intrigants glissements se produisent de l'un aux autres; du reste, ce qui est couramment et abusivement désigné comme le « web 2.0 », ou « web relationnel », se caractérise par la mise en relation de ces différents registres de communication pour des raisons d'abord commerciales. On le sait bien, plus les internautes échangent entre eux, plus ils se donnent à connaître, et plus les données qui leur sont communiquées gagneront en valeur marchande : l'âge du « réseau social » est aussi celui du « profil », c'est-à-dire de l'identification, et donc du ciblage publicitaire. Comme l'écrit acidement Jacob Matthews (2010, 337), « l'usager est "créateur" en ce sens qu'il est aussi - pour ne pas dire avant tout - fournisseur de données marketing, quand bien même sa "production" serait nulle en matière de création écrite, audiovisuelle, musicale ou ludique ». Concernant le cimetière des célébrités, le « web participatif» se dote d'une nouvelle pertinence : les gestes d'activité sur le site pourront valoir comme autant de participation à cet acte de fondation collective que représente le deuil des grands hommes. Jesuismort.com n'échappe pas à la règle du profilage : une série d'actions sont proposées aux « visiteurs » du cimetière qui leur permettront de participer à la pratique commémorative, à condition qu'ils délivrent des données autorisant leur identification. Chercher une tombe, lire une biographie, acheter même un de ces trophées culturels qui alimentent les partenariats commerciaux - tout ceci ne nécessite aucun geste de dévoilement de soi. Toutefois, on peut aller plus loin. Ainsi le site prévoit-il des formes de participation plus étroites à la communauté des « amis des morts » qui se déclinent en différents actes communicationnels. Voici trois formes de contributions graduées. 
Le visiteur à la recherche d'une « tombe » absente se voit proposer de s'inscrire en délivrant son adresse électronique; un courrier lui est alors adressé, qui confirme sa recherche, et s'achève sur cette formule: « JeSuisMort.com vous remercie de participer à l'évolution de www.jesuismort.com ». En l'absence formellement établie du disparu en question, la « recherche de tombe » est enregistrée comme une suggestion et le site formule la promesse suivante : « nous vous contacterons dès publication de la biographie ». Ainsi la participation à l'accroissement de la communauté des morts suppose-t-elle l'inscription au moins numérique dans la communauté des vivants reliés par adresses électroniques. En l'occurrence, notons que le geste même de recherche et de suggestion l'emporte sur l'identité du mort en question : pour tester le dispositif, nous avons suggéré un « mort » qui était encore vivant. La même réponse nous est arrivée que lorsque nous avons cherché un mort vraiment mort. Preuve que l'enregistrement des suggestions s'opère par un architexte automatisé.

Deuxième niveau de participation : le visiteur peut devenir « fan » du site. II s'inscrit alors sur la page consacrée à jesuismort.com sur le réseau Facebook et échange ses données publiées avec les autres membres de ce groupe. Partenariat d'un nouvel ordre finalement qui produit la mise en visibilité sur le bas de page du « cimetière des célébrités 》 des fidèles visiteurs : les visages des vivants s'affichent donc en contrepoint des portraits de disparus.

Figure $6:$ Un exemple de partenariat.

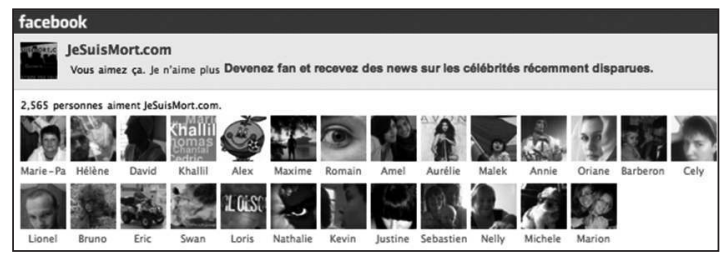

De ce fait, la relation aux morts favorise incontestablement la mise en relation des vivants : sur sa page «Actualités », s'il est inscrit à Facebook, le « fan » reçoit non seulement des nouvelles venues du monde des morts - le 23 juillet 20 I0, il apprend que l'on fête le $6^{\mathrm{e}}$ anniversaire de la disparition de Serge Reggiani - mais aussi, indissociablement, les commentaires que suscitent ces informations du côté des vivants. Ainsi deux « amis » de jesuismort.com dialoguent-ils sur ma « page d'actualités » à propos de ce « grand comédien », échangeant les noms de leurs rôles préférés, et leurs appréciations sur sa disparition regrettée.

Troisième niveau d'interaction, le plus original sans doute: le visiteur se voit proposer de rédiger une lettre au défunt de son choix. «Écrivez une lettre posthume », lit-on en bas de la biographie du mort célèbre : « Votre lettre sera publiée sur le site, mais personne ne vous répondra ». Cet acte de publication mérite d'être analysé pour deux raisons au moins. D'abord, ce qui est présenté 
comme une «lettre posthume» ne ressort du genre épistolaire que par rapprochement métaphorique. Le cadre proposé pour la rédaction se présente surtout comme un courrier électronique, comportant un champ « objet », un champ « lettre »- où la première phrase est déjà pré-écrite, pour encourager l'imitation du style attendu (« Cher Serge Reggiani ») - enfin, un champ « fichier joint ». L'hommage électronique manifeste l'imprégnation des médias informatisés par des modèles textuels et rhétoriques antérieurs, phénomène analysé par Oriane Deseilligny (2006) à propos du journal personnel sur le web. Cette lettre posthume n'est donc ni une lettre, ni posthume : il s'agit d'un message adressé à un destinataire « réel » qui n'est pas le disparu mais les autres visiteurs du site. Une fois la lettre rédigée, un courrier électronique est envoyé à son expéditeur qui lui confirme la publication et lui adresse le lien y afférant. Le texte suit donc un étrange circuit communicationnel. Rédigée par l'internaute selon un cadre instituant fourni par l'architexte, la lettre revient d'abord à son expéditeur, avant d'intégrer un nouveau régime de communication.

Une fois encore, la relation du vivant au mort dérive vers une mise en contact des vivants entre eux. Les lettres sont publiées sur le site et archivées au même titre que les textes biographiques recyclés ou retraités pour alimenter la page de chaque mort célèbre. En guise de commémoration de ces célébrités disparues, le visiteur peut donc choisir de lire les lettres rédigées par d'autres contributeurs - et même, subrepticement, y répondre. Jesuismort.com subit donc ce glissement très familier sur les sites de contribution en ligne qui veut que les échanges sur les contenus deviennent échanges entre contributeurs. Prenons un dernier exemple pour illustrer ce phénomène, révélateur de l'épaisseur des interactions induites par la publication en réseau. Au « top des tops », parmi les 50 morts les plus célèbres des célèbres, figure une étrange image, seule de son espèce. Dans la grille des portraits étagés verticalement selon un classement hiérarchique, se distingue un non-portrait' : une case blanche, occupée en son centre par un point d'interrogation. Cette image absente, ou « visage zéro » (comme il y a, en linguistique, des articles zéro), correspond à l'un des morts les plus commémorés dans le cimetière virtuel : Mahomet.

\footnotetext{
Sur cette question du portrait médiatique, nous nous permettons de renvoyer à notre travail, Face au portrait. Essai sur les représentations de l'individu dans les médias d'actualité, Mémoire d'habilitation à diriger des recherches, Celsa-Paris Sorbonne, 20 I0. Dans cette même livraison, l'article de M.-L. Floréa aborde des problématiques voisines, appliquées au cas des nécrologies de presse.
} 
Figure 7 : L'étagement d'une grille de portraits.

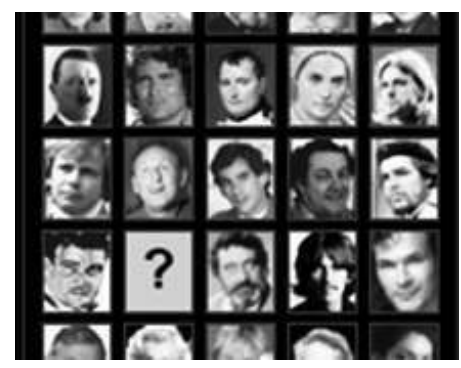

Deux lignes au-dessous d'Adolf Hitler ou de Napoléon Bonaparte, encadré par Bourvil et Marilyn Monroe, le prophète figure, le 23 juillet 20 I0, à la $161^{\text {e }}$ place - bien loin derrière Jacques Brel $\left(n^{\circ} 1\right)$ ou même la mère Denis $\left(8^{e}\right)$. Pourtant, sa place est bien particulière. En effet, 37 lettres, dont la teneur apparaît fort proche des rituels de deuil traditionnels, qui recourent à « une pratique cultuelle de l'écriture » (Urbain, 1989 : 198), lui sont adressées. II s'agit de professions de foi dialogiques où les fidèles se répondent tout en adoptant le ton de la prière : «Paix et salut sur toi ô Mohamed »- tel est le titre choisi pour sa missive par Trayé, de Côte d'Ivoire; Khalid, de son côté, commence sa lettre à Mahomet par une adresse à un contributeur antérieur :

Cher Mahomet,

Je réponds au monsieur qui s'appelle Mathieu franchement vous êtes ignorant avant dearler (sic) de cette façon du grand prophète, lis l'histoire, vous confondez l'islam avec les traditions et les coutumes qui ont été inventées par les musulmans...

Jesuismort.com produit une mise aux normes de tous les morts célèbres, soumis aux impératifs de l'architexte : l'image est prévue par le dispositif, son absence sera donc manifeste, quitte à ce que la culture religieuse entre en conflit avec les attendus de la culture médiatique. De même, les messages d'affection adressés à un chanteur de variété côtoient les expressions du sentiment religieux. À ce titre, dans la lettre de Khalid, le terme de « prophète » appelle un lien commercial qui propose à l'achat l'enregistrement DVD du film réalisé sous ce titre par Mathieu Kassovitz. En retour, cette normalisation des morts racontés autorise l'extrême hétérogénéité des registres sollicités et rend nécessaire l'invention de typologies ad hoc. C'est pourquoi les suggestions des visiteurs du site suscitent aussi la mise en relation des morts entre eux. Les demandes nécrologiques des internautes, et tout particulièrement celles des « fans » du site, finissent par donner lieu à la publication des biographies les plus souvent recommandées. Les morts célèbres ainsi ajoutés à la collection antérieure complètent les différents groupements déjà envisagés. En effet, chaque célébrité voisine virtuellement avec les disparus dont elle partage les « tags » ou index thématiques inscrits en regard de la nécrologie. C'est par cet outil de repérage, qui joue aussi un rôle stratégique dans le référencement par les moteurs de recherche, que se constituent des 
« familles » de morts répondant à d'autres principes de mise en ordre que le simple appel chronologique. Par exemple, Adolf Hitler répond à cinq entrées: « chancelier, criminel de guerre, homme politique, homme d'état, nazi »plus une sixième, placée entre parenthèses - « allemand ». En choisissant l'index « Criminel de guerre », le visiteur découvre le « secteur» réservé par ce cimetière électronique à 4 « criminels de guerre célèbres »: Adolph Hitler y voisine, sans surprise, avec Heinrich Himmler et Hermann Goering, qu'il a, de fait, côtoyés de son vivant, mais aussi avec Amin Dada, issu d'un univers de référence plus éloigné. En revanche, la liste des « hommes d'état » s'avère beaucoup plus étendue : Adolf Hitler y est cité entre Henri IV et Houphouët Boigny.

En vertu des fonctions d'archivage, de classement, d'éditorialisation automatisée rendues possibles par les médias électroniques, la représentation des morts célèbres acquiert sur jesuismort.com une nature dynamique et interactionnelle jamais atteinte auparavant. Dans le vaste processus de laïcisation réglée décrit par Jean-Claude Bonnet (1997: 1847), le site de nécrologie parachève l'entreprise initiée au XIX siècle par un projet encyclopédique tel que le Grand dictionnaire universel de Pierre Larousse, « palmarès des figures de l'histoire », « galerie de silhouettes qui consacre les grands modèles ». Tout se passe comme si les modes de circulation intensifiés des discours sur les morts déterminaient, au bout du compte, un processus de désinstitutionalisation du deuil collectif. Parce que le « tout » du social paraît tangible dans les médiations outillées qu'en proposent les réseaux de mise en relation, ce sont des groupements tout à fait inédits que mettent en visibilité ces classements informatisés de la société des défunts. Reste à s'interroger sur le sens politique d'une telle privatisation de l'hommage post-mortem.

\section{Conclusion}

Qui, du gestionnaire du site, des encyclopédies coopératives, des systèmes de gestions de contenu, des internautes, l'emporte dans le pouvoir pris sur la vie des morts par le cimetière virtuel? Doit-on s'indigner de voir Claude François trôner, intouchable, en première place du «Top des tops »? Faut-il dénoncer les effets pervers des agrégations automatisées qui placent «Vlad III l'empâleur » aux côtés de la Reine Victoria? Les conflits mémoriels ne datent pas de l'internet. Chaque panthéonisation suscite des controverses et, dans l'entre-deux guerres, les héritiers de Victor Hugo menèrent campagne pour que « leur » grand homme quitte le caveau partagé avec Émile Zola dans une proximité qu'ils estimaient avilissante (Wrona, 20l0). Les dictionnaires biographiques expriment toujours le point de vue de leur auteur et sont rarement dépourvus de parti pris idéologiques ou esthétiques. Enfin, les cimetières ne rendent pas toujours hommages aux «bons» morts et la disproportion entre le monument matérialisant l'hommage et l'empreinte collective du disparu peut 
parfois surprendre ou décevoir le visiteur. Jesuismort.com suscite une perplexité d'une nature différente. Qu'il s'agisse de la mise en relation des visiteurs, de la publication des textes biographiques, des «tables » de classement des morts célèbres, une logique dominante, qui jamais ne donne lieu à une franche mise en visibilité, paraît l'emporter. II s'agit d'une logique de comptabilité fondée sur le calcul et rendue possible par l'automatisation. C'est l'architexte qui impose sa loi. Or, lui-même, parce qu'il rend possible le texte, n'est jamais textualisé, ni explicité. Les choix éditoriaux sont imposés par ce qu'il est possible ou non de programmer automatiquement et à faible coût sur un ordinateur. Le gestionnaire du site exclut d'office les contenus trop coûteux - en terme de temps comme en terme financier - si bien que, sans réelle opposition ni résistance, s'impose une culture dominante parce que de coût modeste - ou plutôt de coût modeste, parce que dominante. Claude François, vedette médiatique, champion des ventes hors catégories, a bien sûr beaucoup plus de chance de dominer le «Top 50 des morts célèbres » que Pierre Bourdieu, certes bien placé sur des sites marchands tels qu'Amazon - nouvel outil bio-bibliographique - mais d'une capacité de circulation infiniment inférieure dans le champ ouvert de la culture commune. L'observation parait élitiste; l'enjeu ne consiste pourtant pas à opposer culture populaire et culture des élites, mais à remarquer à quel point, s'agissant des morts comme des vivants, l'industrialisation de la culture passe par celle des textes et de leurs modes de circulation. En confiant à un dispositif automatisé la gestion des stèles dans le cimetière électronique, il est fort probable que l'organisation du deuil se calque sur celle des palmarès de vente, déplaçant le marché de la mort dans des régions qui lui étaient jusque-là étrangères. Rien ne prouve toutefois que ce processus contredise la mise en place de nouveaux rituels commémoratifs. Acheter des objets souvenir ou en conseiller l'achat, recycler des textes lus ailleurs, les commenter et y répondre, sont autant d'actes qui, à leur façon, produisent du social autour de l'écriture des grands morts.

\section{Références}

Ben Amos D., 1997, « Les funérailles de Victor Hugo », pp. 425-464, in : Nora P., dir., Les Lieux de mémoire, T. I, Paris, Gallimard.

Boczkowski P.-J, 20I0, «Ethnographie d'une rédaction en ligne argentine. Les logiques contraires de la production et de l'information chaude et froide », Réseaux, vol. 28, |60-161, janv.-mars, pp. 43-78.

Bonnet J.-Cl., 1997, « Les morts illustres. Oraison funèbre, éloge académique, nécrologie », pp. I831 - 1854, in : Nora P., dir., Les Lieux de mémoire, T. II, Paris, Gallimard.

Dagiral E., Parasie S., dirs, 2010, « Presse en ligne : où en est la recherche? », Réseaux, vol. 28, 160-161, janv.-mars, pp. 15-42.

Davallon J., 2006, Le Don du patrimoine: une approche communicationnelle de la patrimonialisation, Paris, éd. Hermès Sciences/Lavoisier. 
Deseilligny O.,2006, L'Écriture de soi, continuité et mutations. Du cahier aux journaux personnels sur le web (1998-2006), Thèse en sciences de l'information et de la communication, Université Paris X-Nanterre.

Hartog F., 200I, « Plutarque entre les anciens et les modernes », pp. 9-49, in : Vies parallèles de Plutarque, trad. du grec par A.-M. Ozanam, Paris, Gallimard.

— 2003, Les Régimes d'historicité. Présentisme et expérience du temps, Paris, Éd. Le Seuil.

JeanneretY, 2008, Penser la trivialité I. La vie triviale des êtres culturels, Paris, Hermès Sciences/ Lavoisier.

Jeanneret Y, Souchier E., dirs, 1999, « Pour une poétique de l'écrit d'écran », Xoana, 6, pp. 97- 107.

Matthews J., 2010, «Quelques pistes en vue d'une approche critique du web collaboratif », pp. 329-340, in : Millerand F., Proulx S., Rueff J., dirs, Web social. Mutation de la communication, Québec, Presses de l'université du Québec.

Millerand F., Proulx S., Rueff J., dirs, 201 0, Web social. Mutation de la communication, Québec, Presses de l'université du Québec.

Souchier E., 1996, «L'écrit d'écran, pratiques d'écriture et informatique », Communication et langages, 107, I er trim., pp. I05-I 19.

Urbain J.-D., 1989, L'archipel des morts. Cimetière et mémoires en occident, Paris, Payot, 2005.

Wrona A., 2010, « Figures du grand homme », pp. 176-188, in : Pagès A., dir., Zola au Panthéon. L'épilogue de l'affaire Dreyfus, Paris, Presses Sorbonne Nouvelle. 